\title{
Functional Context Awareness: Measuring and Utilizing the Context Dependency of Mobile Usage
}

\author{
Swati S. Gore ${ }^{1}$, Prachi A. Joshi ${ }^{2}$ \\ ${ }^{1}$ Department of Computer Science and Engineering Marthawada Shikshan Prasark Mandal's Deogiri Institute of Engineering \& \\ Management Studies, Aurangabad Maharashtra state, India 2015-16
}

${ }^{2}$ Associate Professor Department of Computer Science and Engineering Marthawada Shikshan Prasark Mandal's Deogiri Institute of Engineering \& Management Studies, Aurangabad Maharashtra state, India 2015-16

\begin{abstract}
Context data brings new open doors for productive and compelling applications and services on mobile devices. An extensive variety of examination has abused context dependency, i.e. the relations in the middle of context(s) and the result, to accomplish huge, evaluated, execution picks up for an assortment of applications and services. These works ordinarily need to manage the difficulties of numerous context sources prompting an inadequate preparing dataset, and the difficulties of energy hungry context sensors. Regularly, they address these difficulties in an application particular and specially appointed way. We free mobile application planners and analysts from these weights by giving a systematic way to deal with these difficulties. Specifically, we 1) characterize and measure the context-dependency of three central sorts of mobile use (went by sites, telephone calls, and application use) in an application freethinker yet viable way, giving understanding into the execution of potential application. 2) Address the test of information meager condition when managing various context sources in an efficient way. 3) Present SmartContext to address the energy challenge via consequently selecting among context sources while guaranteeing a base precision for every estimation. Our examination and discoveries depend on one year of utilization and context follows gathered, in actuality, settings from 24 iPhone clients. We show discoveries with respect to the context dependency of three sorts of mobile utilization from 24 clients, yet our philosophy and the lessons we learn can be promptly stretched out to different sorts of use and additionally framework assets. Our discoveries control the improvement of context mindful frameworks, and highlight the difficulties and assumptions in regards to the context dependency of mobile use.
\end{abstract}

Keywords: Mobile location-based services, security, privacy

\section{Introduction}

Cutting edge mobile frameworks, for example, cell phones and tablets are as of now an essential piece of our lives. They are computationally capable as well as have a rich ability to sense their outside and inside environment. Like the definition by Schilit et al. in [1], we allude to the last referred to state of these situations on the whole as context. Context dependency can be comprehensively characterized as an arrangement of strict or probabilistic tenets and relations in the middle of context(s) and the result [2].

Context has in the past been generally misused to give more usable mobile applications and services, such as substance adjustment [3], [4], client cooperation [5], and data conveyance [6], [7]. Context has additionally been widely misused to give improved framework proficiency and execution, for example, for energy administration [8], [9] and system choice [10]. These outlines misuse the context dependency of mobile use and mobile assets for particular purposes, and show noteworthy, evaluated, execution picks up.

Context mindful frameworks regularly need to manage two principal difficulties. In the first place, managing various wellsprings of context is trying; because of the scourge of dimensionality [11], essentially regarding them as a multidimensional vector results in an inadequate preparing set. Second, liberal use of context can rapidly deplete the devices battery, as some context sensors are amazingly energy hungry. To address the inadequacy test, existing work regularly constrains the quantity of context sources, e.g. to one [8] or two [9], and/or utilize specially appointed or master answers for join various wellsprings of context, e.g. [10]. To address the energy challenge, they frequently utilize impromptu plans along one or a greater amount of these lines: decreasing the recurrence of getting to exorbitant context [12], [13], [14], keeping away from them by and large [10], [12], [13], or substituting them with other context [15], [16], [17], [18].

Specially appointed and application particular methodologies towards these difficulties imply that the originators need to outline and assess another answer for each context-based system. Furthermore, before planning and assessing their application or administration, its creators can just figure its execution result. Our work is freeing in such manner. We give a methodological answer for utilizing different and different wellsprings of context, while dealing with their energy costs. We give a formal yet pragmatic meaning of context dependency, which gives understanding into the execution of applications while remaining application agnostic. We measure the context dependency of three central sorts of mobile utilization utilizing our LiveLab dataset (http://livelab.recg.rice.edu). LiveLab is a phenomenal dataset of reallife context and use follows gathered from 24 iPhone clients more than one year [19]. The mobile utilization we concentrate on are gone by sites, telephone calls, and application usage. 1 We use context data from sensors incorporated with the telephone (i.e. constant clock, Cell ID, Accelerometer, and GPS), and in addition the telephone's last known use state (i.e. application, web, and 


\section{International Journal of Science and Research (IJSR) \\ ISSN (Online): 2319-7064}

Index Copernicus Value (2013): 6.14 | Impact Factor (2014): 5.611

telephone use). Yet, our system and the lessons educated can be reached out to other context and use. Specifically, we make four noteworthy commitments towards evaluating and measuring the context dependency of mobile utilization:

To begin with, in Section 3, we recognize estimation precision in light of most extreme a posteriori (MAP) estimation as an application freethinker yet down to earth, measure for context dependency, interestingly with other hypothetical measurements that are material to multinomial information, for example, entropy as a measure of vulnerability and pseudo $\mathrm{R}$ square as a measure of connection, estimation exactness gives reasonable knowledge into the execution of numerous potential applications, while remaining application rationalist. To permit the effective computation of back probabilities, we present and look at the execution of a few structures ordering and binning of context estimations into a predetermined number of classes, for both ceaseless and discrete context sources. Besides, we address the test of information sparseness when comparing so as to manage with multiple wellsprings of context classifier blend systems.

Second, we introduce a progression of fascinating discoveries with respect to the context dependency of mobile utilization, as takes after:

1) The adequacy of diverse context fluctuates taking into account the use to be assessed, and the quantity of acknowledged reactions. Yet, joining various wellsprings of context reveals their consolidated quality.

2) We find that despite the fact that different context sources are needy, Bayesian mix performs well to combine context data.

3)The context-dependency of use remains generally consistent notwithstanding for lengths of time of one to three months, rather than the full 12 months. This indicates that a littler dataset would be adequate for contextmindfulness.

4)Supervised keeping so as binning can extraordinarily build estimation precision a substantial number of tests in every class or receptacle, while permitting fine embellishment of the canisters.

5)Even however clients are various in their utilization, we can indicate considerable context dependency among every one of them.

Third, we display SmartContext, a system to powerfully or statically improve the expense/precision tradeoffs of context mindfulness, while guaranteeing a base exactness for every estimation occasion. SmartContext exploits the classifier blend calculations we have investigated that have minimal overhead. We demonstrate that by using energy hungry context just at unverifiable times, SmartContext can accomplish an estimation precision inside $1 \%$ of the greatest conceivable exactness, while fundamentally diminishing energy costs by $60 \%$ or more.

Fourth, we exhibit and assess a few specimen applications that profit by context dependency of mobile use. These applications highlight the viable estimation of estimation exactness as a measure of context dependency, and verify the viability of context for evaluating utilization. Our best performing systems, i.e. utilizing Supervised Binning and Bayesian mix, reliably beat regular non-context-based strategies.

\section{Literature survey}

Former work (e.g. [2]) additionally characterize context dependency as an arrangement of strict or probabilistic standards and relations between context (s) and the result. Others outline and execute systems for detecting and handling context data [31],[32]. For a study, see Baldauf et al. [33]. These work bear witness to the criticalness and convenience of context.

Context data, has in the past been generally utilized for particular applications, for example, certain client cooperation (e.g. by Schmidt [5]) and data conveyance (e.g. an update framework by in [6], a traveler guide in [7], and substance adjustment in [3] and [4]). For a study of such cases, see Chen and Kotz [34]. Others have exhibited framework systems in view of context data, e.g. evaluating and anticipating remote system conditions [10], system steering [35], battery administration [8], [36], and energy proficient GPS obligation cycling [12], [13]. Further, Eagle and Pentland have demonstrated that gadget use examples are in reality organized and unsurprising [37]. These outlines and others rely on upon the context dependency of gadget use, and show huge, evaluated, execution picks up by abusing context.

Various other work rely on upon information in regards to utilization to perform. For instance, the work in [10] predicts system conditions to pick the best system interface, yet accept system use is pre-known, despite the fact that it relies on upon the conduct of applications, services, and the client. As another case, the creators of [8] demonstrate that battery utilization is context subordinate. The creators of [9] further research this issue by concentrating on telephone call use, and demonstrate that call terms, and accordingly their energy utilization, are context subordinate. Further, Eagle and Pentland have demonstrated that gadget utilization examples are for sure organized and unsurprising [37]. The value of context has been significant to the point that numerous analysts have outlined and actualized structures for the particular assignment of detecting and preparing context [31], [32], [33]

Our work introduces a methodological answer for utilizing different and different wellsprings of context while dealing with their energy expenses, and displays a formal meaning of context dependency and in addition down to earth routines to figure it. We refrain from concentrating on a solitary application or administration, yet we give functional understanding into the relationship between contextdependency and the execution accomplishments of individual applications.

Various late research have managed decreasing the expense of getting context. These work confirm the test of energy productivity in context mindfulness, yet normally concentrate on single applications and/or static designs. They utilize one

\section{Volume 5 Issue 2, February 2016}




\section{International Journal of Science and Research (IJSR) \\ ISSN (Online): 2319-7064}

Index Copernicus Value (2013): 6.14 | Impact Factor (2014): 5.611

or a greater amount of the accompanying three strategies to decrease energy cost, while holding satisfactory execution. To begin with: recurrence decrease, as in [12], [13], [14] diminishes the testing recurrence of energy hungry context sensors. Second: sensor substitution uses lower energy cost context rather than energy hungry ones, as in [10], [12], [13]. Third: sensor disposal endeavors to use a subset of sensors. We take the third approach in SmartContext, yet not at all like past work that attention on and exploit the properties of a particular application, for example, action identification [15], [16], [18], we give a non specific structure to framework architects to progressively or statically advance the expense/precision exchange offs of context mindfulness. A more broad issue of selecting the best subset of sensors, likewise alluded to as perceptions or indicators, has been the center of many years of exploration in the counterfeit consciousness and operations research groups. These works concentrate on the advancement of data, regularly characterized as either joint entropy or data pick up (delta entropy). It is regular practice to utilize the covetous (nearsighted) arrangement towards this determination issue [40], [38], [39], with ensured execution limits, because of the handling complexities of discovering the ideal arrangement [28], [29], [30]. SmartContext expands upon the insatiable arrangement of Krause and Guestrin [40], however is adjusted to utilizing estimation precision rather than data pick up.

A typical ease sensor utilized for recognizing movement is the accelerometer. With accelerometer as the primary detecting source, movement acknowledgment is generally figured as a classification issue where the preparation information is gathered with experimenters wearing one or more accelerometer sensors in a certain period. Different sorts of classifiers can be prepared and looked at regarding the precision of classification [19, 20, 21 7, 8, 9]. For instance, more than 20 human exercises including strolling, staring at the TV, running, extending thus on can be perceived with genuinely high precision [11]. While it is still not clear what sort of classification calculations works the best, a large portion of the current works require accelerometer sensor(s) to be introduced on pre-identified position(s) close human body.

One approach to make the detecting process less prominent is to utilize the $\mathrm{o}{ }^{\circledR}$-the-rack mobile devices such that no outside sensors are required. A few works have been directed by utilizing the item mobile phones as stages [22, 23, 24, 25, 26, 27]. For instance, CenceMe" [24] empowers individuals from interpersonal organizations to impart their detecting vicinity to theirlbuddies" in a protected way. The framework utilizes the coordinated and in addition outside sensors to catch the clients' status as far as movement, attitude, propensities and environment. A CenceMe model has been made accessible on Facebook, and the execution and assessment of the CenceMe application has additionally been talked about [25]. So also, ISensay" [23] is a context-mindful mobile telephone and uses information from various sources to powerfully change PDA ring tone, ready sort, and in addition decide clients' lun-interruptible" states.

There has been a few examination using telephone logging, e.g. [40], [41], [42], [56]. Contrasted with our follows, they gather extremely restricted context data because of security concerns and battery lifetime confinements. We have defeat these challenges by the cautious configuration and usage of the study, and have gathered extraordinary information.

At long last, there has additionally been impressive exploration on deciding client state from context data e.g. physical action [42]. In this work, we keep away from extricating client state, either specifically or as a break stage, and rather concentrate on the relationship between gadget use and contex.

\section{Proposed system}

In the framework the client who needs to share some data about any location recovers the co ordinates $(x, y)$ of that location from the GPS framework. At that point by utilizing the mystery pivot edge and move, he will change those coordinates say $\left(\mathrm{x}^{\prime}, \mathrm{y}^{\prime}\right)$. A random number generator is utilized to produce the list and is encoded with the mystery key .All the mystery data is passed on to the client's social using so as to gather some safe media like email or telephonic discussion.

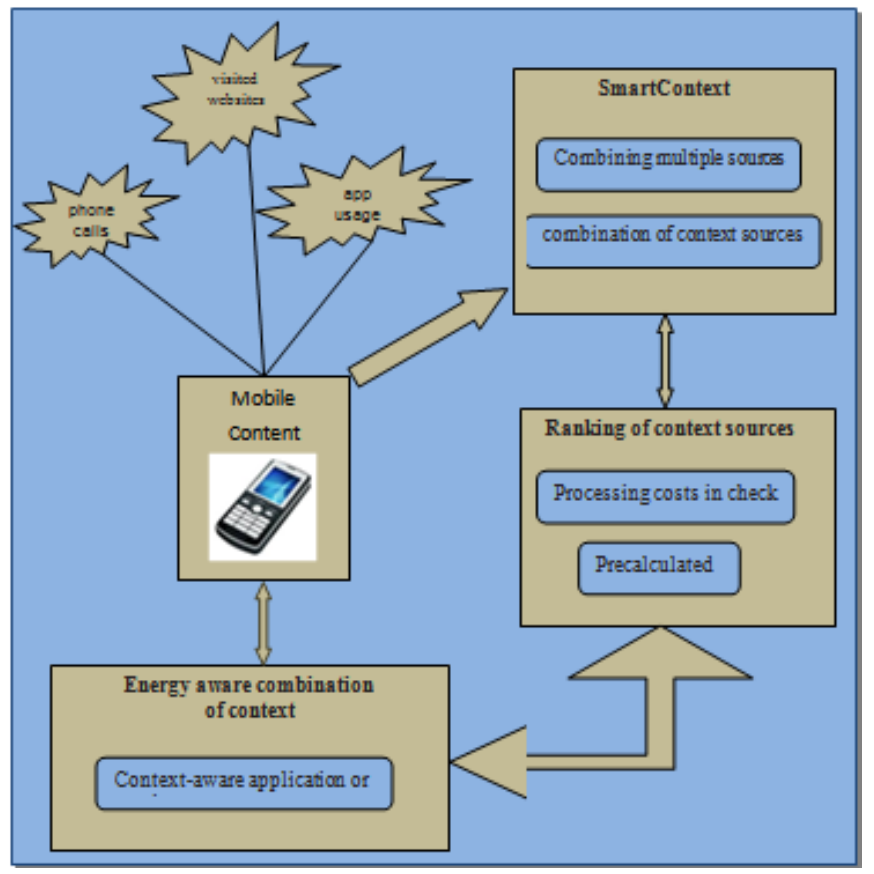

Figure 1: Overview of system operations

\section{SmartContext}

SmartContext, a framework to dynamically or statically optimize the cost/accuracy tradeoffs of context awareness, while ensuring a minimum accuracy for each estimation event. SmartContext takes advantage of the classifier combination algorithms I have explored that have little overhead. We show that by utilizing energy hungry context only at uncertain times,

SmartContext can achieve an estimation accuracy within $1 \%$ of the maximum possible accuracy, while significantly reducing energy costs by $60 \%$ or more. I present and evaluate several sample applications that benefit from context

\section{Volume 5 Issue 2, February 2016}




\section{International Journal of Science and Research (IJSR) \\ ISSN (Online): 2319-7064}

Index Copernicus Value (2013): 6.14 | Impact Factor (2014): 5.611

dependency of mobile usage. These applications highlight the practical value of estimation accuracy as a measure of context dependency, and attest to the effectiveness of context for estimating usage. Our best performing methods, i.e. using Supervised Binning and Bayesian combination, consistently outperform common non-context-based methods.

SmartContext's operation consists of two main steps. The first is determining the ranking of context sources. In order to keep processing costs in check, this ranking must be precalculated, but can be always static, or can be dependent on the context information gained at any step. In the next section we show that a static solution is both practical and performs well. In this case, the ranking needs to be performed only once. The second step is the energy aware combination of context. This has negligible overhead, and is performed dynamically for every estimation event according to the requirements and tradeoffs of the context-aware application or service. Once the ranking is determined, the energy aware combination of context works as follows. For each classification event, SmartContext starts combining multiple sources of context information one by one, in the ordering determined in step one. This can be done with minimum processing overhead, and for any combination of context sources, as explained in below.

\section{Energy aware combination of context}

After running the classifier combination with each additional context source, it checks the criteria of the requesting application or service, for that estimation event. In the evaluation presented here, a fixed minimum estimation accuracy for every estimation event is utilized. However, the application or service may set a different accuracy requirement for each estimation event, or even consider the expected cost of accessing the next context source in determining when to settle with the current estimation accuracy and stop accessing more context sources. SmartContext assures the target estimation accuracy for each estimation event, as long as it is possible to reach that accuracy, while spending no excess cost in acquiring unnecessary context. In other words, in some conditions, no additional costly context is used, while in more uncertain conditions, SmartContext may use up to all the available context sources. The pseudo-code description of SmartContext is shown in Fig. 10.

\section{Combining multiple sources}

Once the ranking is determined, the energy aware combination of context works as follows. For each classification event, SmartContext starts combining multiple sources of context information one by one, in the ordering determined in step one.

\section{Combinations of Context}

By observing the performance of the three types of usage, we can see that combination methods are very useful when a number of meaningful context sources are present. The maxrule consistently outperforms the mean-rule for our data. The mean-rule is known to perform well when classifiers are noisy and highly dependent. Therefore, we conclude that that different context sources are likely not noisy or highly dependent. The surprising fact that Bayes often outperforms other combinations is another indicator that our context sources are either not highly (conditionally) dependent, and/or their dependencies are distributed evenly. On the other hand, even though we use Laplace Correction to reduce the impact of data sparseness, the performance of Bayes is reduced when there are more bins (with fewer samples and therefore more noise). Indeed, it is well known that Bayes is highly susceptible to noise. This is especially notable in web usage. We note that as expected, and as confirmed by the traces (not shown), treating multiple context sources in a multidimensional manner results in virtually no improvement in estimation accuracy. This is unsurprising, as even with only 10 context bins, there were less than 1 percent of samples in any given context. More importantly, most samples belonged to bins that each contained less than 0.1 percent of samples.

\section{Ranking Mobile Context}

SmartContext is based upon the greedy method described in the earlier section, guaranteeing a performance bound of (1 $1=\mathrm{e})$. However, the performance guarantee requires two assumptions. First, the costs of context sources (observations) must be independent from each other. Indeed, mobile context sources typically have independent energy costs, as was our case. Second, the submodularity or diminishing returns property must hold for our utility function (estimation accuracy). While this appears a reasonable assumption, it is necessary to verify it.

It is necessary to show that the estimation accuracy gain resulting from adding (combining) any context source decreases if more context sources were known (combined) beforehand. Note that since SmartContext assumes that free context is always utilized, it is necessary to verify the submodularity only among costly context. shows the estimation accuracy gain for Cell ID, acceleration, and GPS location. Therefore, we conclude that the greedy approach works well for context awareness. In this case, the best ordering is obtainable by ranking the context sources according to their cost effectiveness. In this case, the cost effectiveness of each context source is the marginal estimation accuracy increase divided by its expected energy cost. The expected energy cost can be pre-measured by the system designer, as in our case, or can be measured automatically in software as in. The energy costs of acquiring context on the iPhone 3GS are presented in Table 2. The estimation accuracy of each context source is shown in. The resulting ranking is shown in Table 2 . Note that due to the often significant difference in the energy cost of context sources on mobile devices, their ranking becomes close to the order of their energy cost. Finally, we note that due to the relatively limited number of costly context sources on mobile devices, it is also possible to simply perform a thorough search, calculating the performance of SmartContext under all possible orderings of context sources. For our case, there are three costly context sources, resulting in a total of 3 ! $1 / 46$ possible rankings.

\section{Volume 5 Issue 2, February 2016}




\section{International Journal of Science and Research (IJSR) \\ ISSN (Online): 2319-7064}

Index Copernicus Value (2013): 6.14 | Impact Factor (2014): 5.611

TABLE 2

Ordering \& Measured Energy Cost of Context|

\begin{tabular}{lcc}
\hline Type of context & Order & Energy cost \\
\hline Prior Usage, time\&day & 0 & negligible \\
Accelerometer & 1 & $1.65 \mathrm{~J}$ \\
Cell ID & 2 & $1.2 \mathrm{~J}$ \\
GPS location & 3 & $50-300 \mathrm{~J}$ \\
\hline
\end{tabular}

Figure 2: Ordering and Measures Energy Cost of Context

\section{Conclusion}

We have found that estimation exactness in view of MAP estimation is a down to earth application freethinker measure for context dependency, yet can give understanding into the genuine execution of numerous applications, a few of which are quickly displayed here. These applications bear witness to the viability of context for evaluating usage, and highlight the viable estimation of estimation exactness as the measure of decision for context dependency.

We have additionally found that because of the force law conveyance of usage, assessing mobile usage is extremely testing. Yet, we demonstrate that watchful yet methodological treatment of various wellsprings of context, e.g. combination, discretization, binning, can extraordinarily build estimation exactness. Specifically, we have found that

1) it is important to keep up a sensible number of usage tests in every class, i.e. no under ten, and break even with recurrence discretization of single dimension context accomplishes this.

2) Classifier combination systems can address the information meager condition challenge while using various context sources, and Bayesian combination works best, despite the fact that the contexts are needy. 3) Individualized administered keeping so as to bin incredibly enhances estimation precision a more examples in every receptacle while permitting the fine embellishment of canisters. At long last, despite the fact that the vitality expense of some context sources can be a generous test for context based applications. We address this test through the Smart-Context structure, which guarantees utilizing just as much context sources to meet a base exactness set by the application creator for every estimation occasion. We demonstrate that SmartContext can accomplish an estimation precision inside $1 \%$ of the greatest conceivable, while lessening vitality costs by $60 \%$ or more.

\section{References}

[1] B. Schilit, N. Adams, and R. Want, "Context-aware computing applications," in Proc. Workshop MobilComput. Syst. Appl., 1994, pp. 85-90.

[2] T. Gu, H. K. Pung, and D. Q. Zhang, "A service-oriented middleware for building context-aware services," J. Netw. Comput. Appl., vol. 5, pp. 2656-2660, 2005.

[3] T. Lemlouma and N. Layaida, "Context-aware adaptation for mobile devices," in Proc. Int. IEEE Conf. Mobile Data Manage., 2004, pp. 106-111.

[4] S. K. Kane, J. O. Wobbrock, and I. E. Smith, "Getting off the treadmill:Evaluating walking user interfaces for mobile devices in public spaces," in Proc. 10th ACM Int. Conf. Human Comput. Interaction Mobile Devices Serv., 2008, pp. 109-118.

[5] A. Schmidt, "Implicit human computer interaction through context," Personal Ubiquitous Comput., vol. 4, pp. 191-199, 2000.

[6] N. Marmasse and C. Schmandt, "Location-aware information delivery with commotion," in Proc. 2nd Int. Symp. Handheld Ubiquitous Comput., 2000, pp. 157 171.

[7] K. Cheverst, N. Davies, K. Mitchell, A. Friday, and C. Efstratiou,"Developing a context-aware electronic tourist guide: Some issues and experiences," in Proc. SIGCHI Conf. Human Factors Comput.Syst., 2000, pp. 17-24.

[8] N. Banerjee, A. Rahmati, M. D. Corner, S. Rollins, and L. Zhong,"Users and batteries: Interactions and adaptive energy management in mobile systems," in Proc. Int Conf. Ubiquitous Comput.,2007, pp. 217-234.

[9] N. Ravi, J. Scott, L. Han, and L. Iftode, "Context-aware battery management for mobile phones," in Proc. Int. Conf. Pervasive Comput.Commun., 2008, pp. 224-233.

[10]A. Rahmati and L. Zhong, "Context-based network estimation for energy-efficient ubiquitous wireless connectivity," IEEE Trans. Mobile Comput., vol. 10, no. 1, pp. 54-66, Jan. 2011.

[11]L. Bao and S. S. Intille. Activity recognition from userannotated acceleration data. Pervasive Computing, pages $1\{17,2004$.

[12]Z. Zhuang, K.-H. Kim, and J. P. Singh, "Improving energy efficiency of location sensing on smartphones," in Proc. 8th Int. Conf. Mobile Syst., Appl. Services, 2010, pp. 315-330.

[13] J. Paek, J. Kim, and R. Govindan, "Energy-efficient rateadaptive GPS-based positioning for smartphones," in Proc. 8th Int. Conf. Mobile Syst., Appl. Services, 2010, pp. 299-314.

[14]Y. Wang, B. Krishnamachari, Q. Zhao, and M. Annavaram,"Markov-optimal sensing policy for user state estimation in mobile devices," in Proc. 9th Int. Conf Inform. Process. Sens. Netw,2010, pp. 268-278.

[15]P. Panuccio, H. Ghasemzadeh, G. Fortino, and R. Jafari, "Poweraware action recognition with optimal sensor selection: An adaboost driven distributed template matching approach," in Proc.ACMWorkshop Mobile Syst., Appl., Services Healthcare, 2011, article 5.

[16]P. Zappi, C. Lombriser, T. Stiefmeier, E. Farella, D. Roggen, L. Benini, G. Tr€oster, and R. Verdone, "Activity recognition from on-body sensors: Accuracypower trade-off by dynamic sensor selection," in Proc, 5th Eur. Conf. Wireless Sens. Netw., 2008, pp. 17-33.

[17] M. Annavaram, N. Medvidovic, U. Mitra, S. Narayanan, D. Spruijt-Metz, G. Sukhatme, Z. Meng, S. Qiu, R. Kumar, and G. Thatte. Multimodal sensing for pediatric obesity applications. In International Workshop on Urban, Community, and Social Applications of Networked Sensing Systems (UrbanSense08), November 2008.

[18] Y. Wang, J. Lin, M. Annavaram, Q. A. Jacobson, J. Hong, B. Krishnamachari,and N. Sadeh, "A framework of energy efficient mobile sensing for automatic user state recognition," in Proc. $7^{\text {th }}$ Int. Conf. Mobile Syst., Appl. Serv., 2009, pp. 179-192.

\section{Volume 5 Issue 2, February 2016




\section{International Journal of Science and Research (IJSR) \\ ISSN (Online): 2319-7064}

Index Copernicus Value (2013): 6.14 | Impact Factor (2014): 5.611

[19] T. Choudhury, G. Borriello, S. Consolvo, D. Haehnel, B. Harrison, B. Hemingway, J. Hightower, P. Klasnja, K. Koscher, An. LaMarca, J. A. Landay, L. LeGrand, J. Lester, A. Rahimi, A. Rea, and D. Wyatt. The mobile sensing platform: An embedded activity recognition system. Pervasive Computing, 7(2):32\{41, 2008.

[20] N. D. Lane, H. Lu, S. B. Eisenman, and A. T.Campbell. Cooperative techniques supporting sensor-based people centric inferencing. Lecture Notes in Computer Science, 5013/2008:75\{92, 2008.

[21]P. Zappi, T. Stiefmeier, E. Farella, D. Roggen, L. Benini, and G. Troster. Activity recognition from onbody sensors by classi ${ }^{-}$er fusion: sensor scalability and robustness. In 3rd International Conference on Intelligent Sensors, Sensor Networks and Information, (ISSNIP 2007), 2007.

[22] J. Burke, D. Estrin, M. Hansen, A. Parker, N. Ramanathan, S. Reddy, and M. B. Srivastava. Participatory sensing. In WSW'06 at SenSys '06, Boulder, Colorado, USA, 2006.

[23]D. Siewiorek, A. Smailagic, J. Furukawa, N. Moraveji, K. Reiger, and J. Sha®er. Sensay: A context-aware mobile phone. pages 248\{249. IEEE Computer Society, 2003.

[24] E. Miluzzo, N. D. Lane, S. B. Eisenman, and A. T. Campbell. Cenceme - injecting sensing presence into social networking applications. In Gerd Kortuem, Joe Finney, Rodger Lea, and Vasughi Sundramoorthy,editors, EuroSSC, volume 4793 of Lecture Notes in Computer Science, pages 1\{28. Springer, 2007.

[25]E. Miluzzo, N. Lane, K. Fodor, R. Peterson, S. Eisenman, H. Lu, M. Musolesi, X. Zheng, and A. Campbell. Sensing meets mobile social networks: The design, implementation and evaluation of the cenceme application. In Proceedings of ACM SenSys 2008, November 2008.

[26] S. Gaonkar, J. Li, R. R. Choudhury, L. Cox, and A. Schmidt. Micro-blog: sharing and querying content through mobile phones and social participation. In MobiSys '08: Proceeding of the 6th international conference on Mobile systems, applications, and services, pages $174\{186$, New York, NY, USA, 2008.ACM

[27]E. Welbourne, J. Lester, A. LaMarca, and G. Borriello. Mobile context inference using low-cost sensors. In Workshop on Location- and Context-Awareness (LoCA 2005), Boulder, Colorado,USA, 2005.

[28] C.-W. Ko, L. Jon, and M. Queyranne, "An exact algorithm for maximum entropy sampling," Operations Res., vol. 43, pp. 684-691, 1995.

[29]B. Goldengorin, G. A. Tijssen, and M. Tso, "The maximization of submodular functions: Old and new proofs for the correctness of the dichotomy algorithm," Univ. of Groningen, Groningen, Netherlands Research Report 99A17, Res. Inst. SOM (Syst., Org.Manage.), 1999.

[30]A. Krause and C. Guestrin, “Optimal nonmyopic value of information in graphical models-efficient algorithms and theoretical limits," in Proc. Int. Joint Conf. Artif. Intell., 2005, pp. 1339-1345.
[31]H. van Kranenburg, M. S. Bargh, S. Iacob, and A. Peddemors, "A context management framework for supporting context-aware distributed applications," IEEE Commun Mag., vol. 44, no. 8,pp. 67-74, Aug. 2006.

[32] Y. Lee, S. S. Iyengar, C. Min, Y. Ju, S. Kang, T. Park, J. Lee, Y. Rhee, and J. Song, "MobiCon: A mobile contextmonitoring platform,"Commun. ACM, vol. 55, pp. 5465, 2012.

[33] M. Baldauf, S. Dustdar, and F. Rosenberg, "A survey on contextaware systems," Int. J. Ad Hoc Ubiquitous Comput., vol. 2, pp. 263-277, 2007.

[34] G. Chen and D. Kotz, "A survey of context-aware mobile computing research," Dept. of Computer Science, Dartmouth College,Hanover, NH, Tech. Rep. TR2000-381, 2000.

[35] G. Cugola, M. Migliavacca, U. Roedig, and C. Sreenan, "A context and content-based routing protocol for mobile sensor networks,"in Proc. 6th Eur. Conf. Wireless Sens. Netw., 2009, pp. 69-85.

[36] N. Ravi, J. Scott, L. Han, and L. Iftode, "Context-aware battery management for mobile phones," in Proc. 6th Annu IEEE Int. Conf. Pervasive Comput. Commun., 2008, pp. 224-233.

[37] N. Eagle and A. Pentland, "Eigenbehaviors: Identifying structure in routine," Behavioral Ecology Sociobiology, vol. 63, pp. 1057-1066,2009.

[38]L. Van Der Gaag and M. Wessels, "Selective evidence gathering for diagnostic belief networks," AISB Quarterly, vol. 86, pp. 23-34,1993.

[39] S. L. Dittmer and F. V. Jensen, "Myopic value of information in influence diagrams," in Proc. 13th Conf. Uncertainty Artif. Intell.,1997, pp. 142-149.

[40] J. Froehlich, M. Y. Chen, S. Consolvo, B. Harrison, and J. A.Landay, "My experience: A system for in situtracing and capturing of user feedback on mobile phones," in Proc. 5th Int. Conf. Mobile Syst., Appl. Serv., 2007, pp. 57-70.

[41]N. Eagle and A. Pentland, "Reality mining: Sensing complex social systems," Personal Ubiquitous Comput., vol. 10 , pp. 255-268,2006.

[42] C. V. C. Bouten, K. T. M. Koekkoek, M. Verduin, R. Kodde, and J. D. Janssen, "A triaxial accelerometer and portable data processing unit for the assessment of daily physical activity," IEEE Trans. Biomed. Eng., vol. 44, no. 3, pp. 136-147, Mar. 1997. 